\title{
Hybrid Analog-Digital Transmit Beamforming for Spectrum Sharing Backhaul Networks
}

\author{
Miguel Ángel Vázquez, Luis Blanco and Ana I. Pérez-Neira, Senior Member, IEEE
}

\begin{abstract}
Next generation wireless backhauling networks are meant to share the same spectrum resources in order to deal with the exponential base station data rate demands. One alternative is to consider a very aggressive frequency reuse among backhaul links and implement interference mitigation techniques. This paper deals with the problem of analog-digital transmit beamforming under spectrum sharing constraints for backhaul systems. In contrast to fully-digital designs where the spatial processing is done at baseband unit with all the flexible computational resources of digital processors, analogdigital beamforming schemes require that certain processing is done through analog components, such as phase-shifters or switches. These analog components do not have the same processing flexibility as the digital processor but; on the other hand, they can substantially reduce the cost and complexity of the beamforming solution. Precisely, with an hybrid analog-digital scheme the number of radiofrequency chains can be reduced by extending the processing through the analog part and; therefore, reducing the overall cost and digital bandwidth requirements. This work presents the joint optimization of the analog and digital parts that results in a non-convex, NP-hard and coupled problem. In order to solve it, an alternating optimization with a penalized convex-concave method is proposed. According to the simulation results, this novel iterative procedure is able to find a solution that behaves close to the fully-digital beamforming upper bound scheme. All in all, despite the computational complexity of the proposed scheme is relatively high, it is adequate for backhauling networks where nodes are static and the beamforming weights do not need to be updated on a frame basis.
\end{abstract}

\section{INTRODUCTION}

In the forthcoming $5 \mathrm{G}$ broadband communications, flexible and high throughput backhauling systems are mandatory. Although wireless backhaul solutions are currently deployed in suburban and rural areas, future wireless backhauling systems will have a key role in the overall $5 \mathrm{G}$ ecosystem. This fact requires that the nowadays fixed wireless backhaul links shall be re-thought. Indeed, current solutions cannot quickly react to dynamic traffic demands by reconfiguring the network topology and, as a consequence, the nowadays equipment is required to be upgraded with pointing capabilities (beamforming) in order to meet $5 \mathrm{G}$ expectations [2].

In addition, due to the tremendous increase of the user data rate demands, very efficient spectrum allocation policies will

This work has received funding from the European Union's Horizon 2020 research and innovation programme under grant agreement No 645047 (SANSA); the Spanish Ministry of Economy and Competitiveness (Ministerio de Economia y Competitividad) under project TEC2014-59255-C3-1-R (ELISA); and from the Catalan Government (2014SGR1551). The material in this paper has been partially presented at IEEE SPAWC 2016 [1].

M. Á. Vázquez and Luis Blanco are with Centre Tecnològic de les Telecomunicacions de Catalunya (CTTC), Barcelona, Spain. Ana I. PérezNeira is with Universitat Politecnica de Catalunya (UPC) and CTTC.

Emails: mavazquez@cttc.es, lblanco@cttc.es, ana.perez@cttc.es become mandatory. As result, current per-link fixed licensing will become obsolete and next generation backhauling systems are meant to share the available spectrum.

Backhauling systems are generally deployed in the millimeter wave (mmWave) bands (e. g. 18 or $28 \mathrm{GHz}$ ). Spectrum sharing studies in the mmWave bands have been recently presented for the cellular access communication in [3]-[5]. According to these results, despite the harness of directive antennas used in the mmWave bands, interference between the different agents severely impacts the achievable data rates. Consequently, interference mitigation techniques become mandatory in order to keep the achievable rates high.

This paper considers the scenario described in [5], where a primary operator decides to share with a secondary operator its available spectrum devoted to its backhauling network. This secondary operator has to maintain the interference generated to the primary operator below a certain value. Precisely, it is compulsory that the backhaul links are equipped with multiple antennas in order to control the tentative interference to the primary operator while maximizing the data rate of the intended link. This latter requirement leads to a different multiantenna design compared to the growing literature in mmWave multiantenna solutions where the secondary spectrum sharing licensing aspects are not treated.

Focusing on the single transmitter case in presence of a single intended receiver and multiple non-intended ones, the sub-6 GHz fully-digital case has been studied in [6]. We cannot mimic this design for mmWave as fully-digital beamforming designs cannot be implemented due their cost and complexity. Indeed, attending to the cost-performance trade-off, the most adequate multiantenna architecture is the hybrid analog-digital [7], where an analog beamforming network (BN) processes the signals received by the $Q$ antennas into a set of $N_{R F} \leq$ $Q$ radiofrequency (RF) chains. Although this $\mathrm{BN}$ can ideally connect every RF chain with each antenna with amplitude and phase control, low-complexity analog architectures with only phase control result in a large complexity reduction [7]. The aim of this paper is to revisit the fully-digital solution transmit beamforming in [6] and adapt it to the hybrid analog-digital design.

For the $\mathrm{BN}$ we consider different types of architectures; namely, fully-connected, interleaved and localized. While the fully-connected is an ideal architecture which leads to a very costly analog design (i.e. each RF chain shall be connected with each antenna), interleaved and localized architectures provide a reasonable cost-complexity trade-off. For each RF chain to antenna connection, we assume that the analog processing is done either via phase shifters or switches. For 
each case, the optimization problem substantially differs to each other and it shall be reconsidered. Precisely, while the processing done via phase shifters requires the optimization of a set of complex vectors with unit norm entries, in case we consider switches as analog components, it requires a binary optimization.

Interference mitigation in hybrid analog-digital beamforming techniques are treated in multiuser scenarios [8]-[11]. Nevertheless, among the aforementioned works, the problem considered in this paper is not addressed. Here, we consider the maximization of the array gain in a single direction while maintaining the interference generated to the primary network receivers under a certain threshold $\epsilon$. This problem has been originally proposed in [1] where an optimization method based on a non-smooth technique [12] is presented. In addition, the work in [13] studies the interference mitigation case when a fully-connected $\mathrm{BN}$ is assumed with a multiple-input-multipleoutput (MIMO) communication.

This paper extends the contribution in [1], [13] and the current hybrid analog-digital beamforming solutions for interference mitigation since our study contemplates any arbitrary connectivity matrix and a either phase shifters or switches analog components. This approach is different from the current beamforming designs. In [14] an optimization scheme for nulling using a localized arrays is proposed. Our proposal considers a perfect control of the interference power for different sub-array architectures. Moreover, sub-array sparse nulling optimization can be found in [15]. Again, our proposed scheme is able to smartly control the interference of a larger number of hybrid architectures.

In addition, current hybrid analog-digital MIMO processing schemes are based on the Frobenius norm minimization between the fully-digital case and the hybrid one [8], [16][20]. In contrast to this approach, in this paper we tackle the problem directly, which guarantees the fulfilment of the interference power limit constraints. These restrictions might be violated whenever the minimum Frobenius norm approach is elected (i.e. the resulting beamforming approximation might violate the interference constraints). Additionally, the presented work considers the per-antenna power constraints whereas in the aforementioned solutions sum-power constraint is assumed. As a matter of fact, optimizing a multi-stream precoding matrix is a more challenging problem than the one studied in this paper of single-stream communication. In any case, the open problems reported in [21] (i.e. partiallyconnected with phase shifters and any arbitrary connection with switches) are still relevant for the single-stream transmission.

The proposed method consists of an alternating optimization problem which sequentially optimizes the analog and the digital part respectively. While the digital part can be cast as a convex optimization problem, the analog part is a NP-hard non-convex problem. For the analog design, even the semidefinite relaxation (SDR) followed by a randomization method fails in delivering an efficient solution. This is, the relaxed problem yields to a high-rank solution and the randomization method is unable to provide an efficient rank-one solution due to the equality constraints. This fact is also reported in [12] and the solution is mimicked in [1] for the considered optimization problem.

In contrast to the authors previous approach in [1] in here we use the penalty convex concave procedure (PCCP) [22] for optimizing the analog part. This novel method solves a nonconvex quadratically constraint quadratic program (QCQP) guaranteeing that the constraints are not violated. Remarkably, PCCP is able to deal with not only the equality constraints used when assuming phase shifters, but also the binary constraints when considering switches as analog components.

We compare the proposed alternate scheme with the fullydigital beamforming with per-antenna power constraints and it is observed that very close achievable rates are obtained. Concretely, the simulation results show that different sub-array schemes with different implementation complexities lead to very similar results.

To sum up, the contributions of this work are:

1) We propose an optimization method for obtaining hybrid analog-digital beamforming designs in spectrum sharing scenarios. This problem was treated before the for fullydigital case in [6], for the all-analog with phase shifters in [23] and for the fully-connected with phase shifters analog components BN MIMO case in [13].

2) The method admits any arbitrary connectivity matrix but we focus on three: fully-connected, interleaved and localized. While for fully-connected and localized there are some recent results for the general MIMO processing scheme with phase shifters analog components [8], [16][20], none of them consider the per-antenna power constraints as this paper focuses on.

3) Switches and phase shifters can be used with our approach. When switches are used, this work extends [20], where a fully-connected array with total power constraints is considered for solving the MIMO processing problem. Precisely, we consider an arbitrary connectivity matrix and per-antenna power constraints.

4) In contrast to current approaches, this work considers the direct hybrid analog-digital precoding optimization instead of minimizing the Frobenius norm between the fully-digital and hybrid designs. This is of great importance as the interference power limits might be violated in case there are no used as a constraint.

The rest of the paper is organized as follows. Section II presents the system model and the different beamforming architectures. Section III describes the optimization problems to be solved. Section IV proposes and alternating projection method for dealing with the hybrid analog-digital optimization. Section V presents the numerical results. Finally, conclusions are presented in Section VI.

Notation: Throughout this paper, the following notations are adopted. Boldface upper-case letters denote matrices and boldface lower-case letters refer to column vectors. $(.)^{H}$, $(.)^{T},(.)^{*}$ and $(.)^{+}$denote a Hermitian transpose, transpose, conjugate and diagonal (with positive diagonal elements ) matrix, respectively. $\mathbf{I}_{N}$ builds $N \times N$ identity matrix and $\mathbf{0}_{K \times N}$ refers to an all-zero matrix of size $K \times N$. If $\mathbf{A}$ is a $N \times N$ matrix. $[\mathbf{X}]_{i j}$ represents the ( $i$-th, $j$-th) element of matrix $\mathbf{X} . \otimes$, ○ and $\|$.$\| refer to the Kronecker product,$ 
the Hadamard product and the Frobenius norm, respectively. Vector $\mathbf{1}_{N}$ is a column vector with dimension $N$ whose entries are equal to 1 . vec $(\cdot)$ denotes the vectorization operator. $\mathcal{I}\{\cdot\}$ and $\mathcal{R}\{\cdot\}$ denote the imaginary part and real part operators, respectively. $[\mathbf{x}]_{q}$ denotes the $q$-th component of the $\mathbf{x}$ vector.

\section{System Model AND Hybrid ARChitectures}

\section{A. System Model}

Let us assume a secondary backhaul link operating at the mmWave band in presence of a primary backhauling network. We assume that the transmitter is equipped with $Q$ antennas and the receiver with $M$, the received signal of the secondary user can be modelled as

$$
y=\sqrt{P} \gamma_{s} \mathbf{u}^{H} \mathbf{H} \mathbf{v} s+n,
$$

where $\mathbf{v} \in \mathbb{C}^{Q \times 1}$ is the transmit beamforming vector, $P$ is the transmit power, $\mathbf{H} \in \mathbb{C}^{M \times Q}$ is the channel matrix, $\mathbf{u} \in$ $\mathbb{C}^{M \times 1}$ the receive beamforming vector, $\gamma_{s}$ is the path-loss between the transmitter and the intended receiver and $n$ is the zero-mean unit-variance additive white Gaussian noise. The transmitter sends an unit-norm symbol which is denoted by $s$.

Similarly to the secondary user, we assume there are a set of $K$ primary interfered users equipped with $R_{k}$ for $k=1, \ldots, K$ receive antennas. The received signal by the $k$-th non-intended primary receiver can be modelled as

$$
z_{k}=\sqrt{P} \gamma_{p, k} \mathbf{t}_{k}^{H} \mathbf{G}_{k} \mathbf{v} s+o_{k}, \quad k=1, \ldots, K,
$$

where $\mathbf{t}_{k} \in \mathbb{C}^{R_{k} \times 1}$ is the receive beamforming of the $k$ th primary user, $\mathbf{G}_{k} \in \mathbb{C}^{R_{k} \times Q}$ the channel matrix between the secondary transmitter, $\gamma_{p, k}$ is the path-loss between the transmitter and the $k$-th primary receiver and $o_{k}$ is the zeromean and unit-variance additive white Gaussian noise.

We consider the narrowband mmWave channel model [24], [25] which can be modelled as

$$
\mathbf{H}=\frac{1}{\sqrt{L}} \sum_{c=1}^{C} \sum_{l=1}^{L} \alpha_{c l} \mathbf{a}_{r x}\left(\theta_{c l}^{r x}\right) \mathbf{a}_{t x}\left(\theta_{c l}^{t x}\right)^{H},
$$

where $L$ denote the number of sub-paths and $C$ the number of clusters. The value $\alpha_{c l}$ is a small scale fading term of the $l$-th sub-path at the $c$-th cluster for $c>1$ and $l>1$ otherwise, for the value $\alpha_{11}$ is assumed to be equal to one. Vectors $\mathbf{a}_{t x}(\cdot)$ and $\mathbf{a}_{r x}(\cdot)$ are the antenna array responses of both the transmitter and the receiver respectively. The transmit and receive antenna array responses depend on both the angles of departure (AoD), $\theta_{c l}^{t x}$, and angles of arrival (AoA), $\theta_{c l}^{r x}$, respectively.

The steering vector a depends on the antenna array structure and the element spacing. In the following, we consider an uniform linear array (ULA) whose steering vector can be written as

$$
\mathbf{a}_{\mathrm{ULA}}(\theta)=\frac{1}{\sqrt{Q}}\left(1, e^{j \frac{2 \pi}{\lambda} d \sin (\theta)}, \ldots, e^{j \frac{2 \pi}{\lambda}(Q-1) d \sin (\theta)}\right),
$$

where $Q$ is the number of antenna elements, $d$ the element spacing and $\lambda$ the wavelength.

Finally, $\gamma_{k}$ for $k=0, \ldots, K$ denotes the path-loss which can be written as

$$
\gamma=\left(\frac{1}{4 \pi l \lambda}\right)
$$

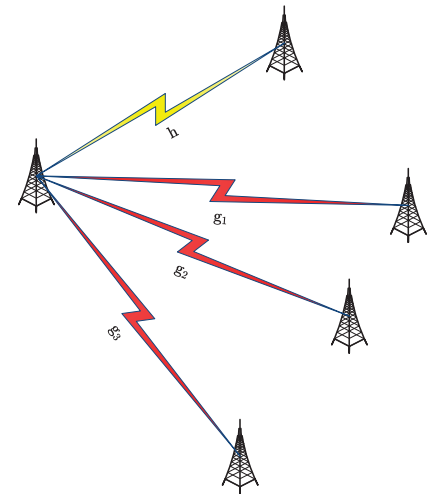

Fig. 1: Spectrum sharing backhauling network. The transmitter shares information with a single receiver (yellow line) in presence of interfered secondary users (red lines).

where $l$ is the distance between the transmitter and the receiver.

In the overall paper we consider a backhauling scenario according to the 'above the roof top' channel model presented in [25]. This is further described in the numerical results Section.

It is important to remark that we assume that the receive beamforming vectors of both the secondary and primary receivers are fixed and known by the transmitter. For notational convenience, we consider the following definitions

$$
\begin{gathered}
\mathbf{h}^{T}=\mathbf{u}^{H} \mathbf{H}, \\
\mathbf{g}_{k}^{T}=\mathbf{t}_{k}^{H} \mathbf{G}_{k} \quad k=1, \ldots, K .
\end{gathered}
$$

Figure 1 depicts an example of the backhauling spectrum sharing scenario.

The objective of this paper is to design $\mathbf{v}$ so that it maximizes the array gain to the secondary receiver

$$
\left|\mathbf{h}^{H} \mathbf{v}\right|^{2}
$$

while keeping the array gain to the non-intended receivers

$$
\left|\mathbf{g}_{k}^{H} \mathbf{v}\right|^{2} \quad k=1, \ldots, K,
$$

below a certain threshold.

Prior to formalizing the optimization problem for the different hybrid analog-digital beamforming architectures, the following Section describes them.

\section{B. Hybrid Analog-Digital Antenna Array Architectures}

Hybrid analog-digital schemes have been used in the past for both radar and communication systems. These types of beamforming structures have two separate processing parts at the analog and at the digital domain. While the digital processing is benefited from the use of all the computational resources, the analog processing is done with RF components. These components are usually phase shifters or switches. Both the phase shifters and the switches lead to different processing restrictions. While a phase shifter only controls the phase of a given RF signal the switch either connects or disconnects 


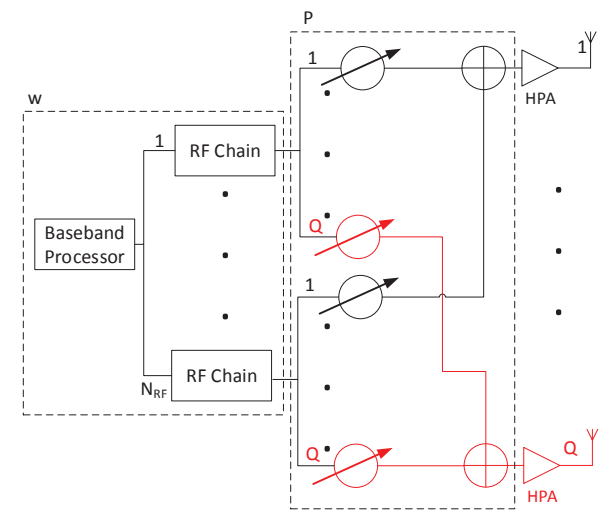

Fig. 2: Fully-connected hybrid analog-digital beamformer using phase shifters or switches.

a RF chain to an antenna. The switching operation can be modelled as a binary variable and the phase shifter as a unitnorm complex variable.

The hybrid analog-digital beamforming solutions present differences depending on how the connections between the RF chain and the antenna are performed. As a general statement, each RF chain of the digital part is connected with one or more antenna through an analog component. The most complex scheme is the one that considers an all-to-all connection (i.e. each RF chain is connected to all antennas through an analog component). Figure 2 presents a fully-connected scheme with phase shifters. From the figure it can be observed that it is required $Q N_{R F}$ connection lines and components.

In order to substantially reduce the number of connections and analog components other connection matrices are conceived. In the rest of the paper we will consider two of them; namely, localized and interleaved. Whereas a localized architecture connects each RF chain a subset of sequential antennas, the interleaved scheme interconnects the different RF chains with separated antennas. As the connection lines are longer in an interleaved scheme than in the localized one, the implementation complexity and losses are higher. Figure 3 shows both interleaved and localized schemes with phase shifters analog components, which can be substituted by switches.

Remarkably, each scheme has a different cost and implementation complexity. The evaluation of the scheme that offers the best cost-performance trade-off is out of the scope of this paper and it is left for further works. Furthermore, each scheme presents different implementation losses however, they are not considered in this paper and ideal analog components are assumed.

\section{BeAmforming Optimization Problem Statement}

This section presents the optimization problems in order to allow the spectrum sharing coexistence with the hybrid analogdigital beamforming configurations presented in the previous

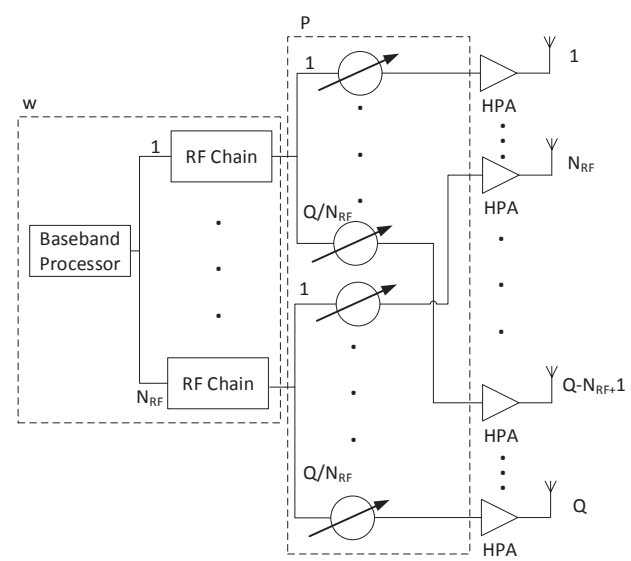

(a) Interleaved hybrid analog-digital beamformer using phase shifters or switches.

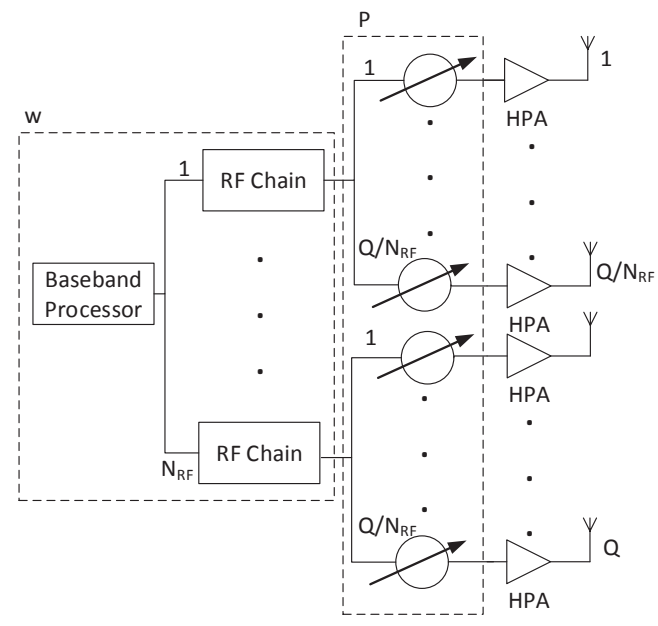

(b) Localized hybrid analog-digital beamformer using phase shifters or switches.

Fig. 3: On the right it is described a localized beamforming scheme while on the left figure can be observed an interleaved implementation. In both cases the analog component is a phase shifter although it can also be a switch.

section. Precisely, we present the different optimization problems to be addressed depending on the underlying analogdigital beamforming architecture and components. For the sake of completeness and since its performance is considered as a benchmark, the digital beamforming optimization is presented in the following section as well.

\section{A. Digital Beamforming}

In digital beamforming the spatial processing is done at the baseband processor. This is, the transmit signal is multiplied by a complex vector before being delivered to the digital-toanalog converters. As the processing is done in the digital domain, it can perform a large number of flexible operations at expenses of costly RF chains and very high performance 
processors.

The transmit beamforming optimization with a fully-digital architecture can be mathematically described as follows

$$
\begin{aligned}
& \underset{\mathbf{v}}{\operatorname{maximize}}\left|\mathbf{h}^{H} \mathbf{v}\right|^{2} \\
& \text { subject to } \\
& \left|\mathbf{g}_{k}^{H} \mathbf{v}\right|^{2} \leq \epsilon_{k} \quad k=1, \ldots, K, \\
& \left|[\mathbf{v}]_{i}\right|^{2} \leq 1 / Q \quad i=1, \ldots, Q .
\end{aligned}
$$

The values $\left\{\epsilon_{i}\right\}_{i=1}^{K}$ are known by the transmitter. Note that we have considered per-antenna power constraints so that each of them have a maximum available power of $\frac{1}{Q}$. The optimization problem (10) is a QCQP that admits a convex reformulation. Bearing in mind the derivations in [6] for total power constraint, the optimization problem for perantenna power constraints can be solved with the following optimization method

$$
\begin{aligned}
& \underset{\mathbf{v}}{\operatorname{maximize}} \mathcal{R}\left\{\mathbf{h}^{H} \mathbf{v}\right\} \\
& \text { subject to } \\
& \mathcal{I}\left\{\mathbf{h}^{H} \mathbf{v}\right\}=0, \\
& \left|\mathbf{g}_{k}^{H} \mathbf{v}\right|^{2} \leq \epsilon_{k} \quad k=1, \ldots, K, \\
& \left|[\mathbf{v}]_{i}\right|^{2} \leq 1 / Q \quad i=1, \ldots, Q .
\end{aligned}
$$

The optimization problem (11) can be cast as a second order cone program (SOCP) which is convex and; thus, it can be efficiently solved via interior point methods.

\section{B. Hybrid Analog-Digital Beamforming}

In case we consider an hybrid scheme with a phase shifter network, both the analog $\mathrm{BN}$ and the digital processing part shall be optimized. Let us denote $\mathbf{P} \in \mathbb{C}^{Q \times N_{\mathrm{RF}}}$ the analog processing part and $\mathbf{w} \in \mathbb{C}^{N_{R F} \times 1}$, the digital part. The joint optimization problem of $\mathbf{w}$ and $\mathbf{P}$ can be written as

$$
\begin{aligned}
& \underset{\mathbf{w}, \mathbf{P}}{\operatorname{maximize}}\left|\mathbf{h}^{H} \mathbf{P} \mathbf{w}\right|^{2} \\
& \text { subject to } \quad\left|\mathbf{g}_{k}^{H} \mathbf{P} \mathbf{w}\right|^{2} \leq \epsilon_{k} \quad k=1, \ldots, K, \\
& \left|[\mathbf{P w}]_{q}\right|^{2} \leq 1 / Q \quad q=1, \ldots, Q, \\
& \mathbf{P} \in \mathcal{P}
\end{aligned}
$$

where $\mathcal{P}$ depends on both the connectivity matrix and whether we assume phase shifters or switches.

It is important to remark that the optimization problem in (12) is a novel approach and constitutes a very challenging problem. Even though the expression is a close-to-real problem, from the best of authors knowledge, it has not been addressed previously in the literature apart from the work in [1]. Other works like those presented in [16]-[18] only consider the fully-connected analog part with phase shifters or switches [19]. Neither the mentioned works nor [8], [20] where the localized scheme with phase shifters is treated, per-antenna power constraints as (12c) depicts are considered. To sum up, although the aforementioned works deal with the difficult problem of MIMO precoding, none of them considered either such a variety of analog components or the per-antenna power constraints as this paper proposes.
To the best of authors knowledge, the scheme in [26] is the only one that considers the per-antenna power constraints as we consider in this paper for the fully connected architecture with phase shifters. However, in the numerical simulation results Section is shown that [26] is not capable of delivering an efficient solution as the interference constraints are not guaranteed to be preserved with this method.

As elegantly reported in [21] for the MIMO case, depending on $\mathcal{P}$ the optimization problem can become intractable for the known convex optimization techniques. In this paper we provide convex relaxations to the proposed architecture and analog components.

In case phase shifter analog components are used, we consider the following $\mathcal{P}$ :

$$
\begin{aligned}
& \mathcal{P}_{\text {full-PS }}:\left|[\mathbf{P}]_{m, n}\right|^{2}=1, \\
& \mathcal{P}_{\text {interleaved-PS }}:\left|[\mathbf{P}]_{m, n}\right|^{2}=\left[\mathbf{1}_{\kappa} \otimes \mathbf{I}_{N_{R F}}\right]_{m, n}, \\
& \mathcal{P}_{\text {localized-PS }:}:\left|\left[\mathbf{P}_{m, n}\right]\right|^{2}=\left[\mathbf{I}_{N_{R F}} \otimes \mathbf{1}_{\kappa}\right]_{m, n}, \\
& \text { for } m=1, \ldots, Q \quad n=1, \ldots, N_{\mathrm{RF}} \text { and } \\
& \kappa=\frac{Q}{N_{R F}},
\end{aligned}
$$

which is assumed to be an integer value. Remarkably, as stated in [8] when $N_{R F} \geq 2$ and (13) is considered, the optimization problem in (12) admits a trivial solution based on the optimal fully-digital design. However, for the other connectivity schemes the optimal solution of (12) is an open problem.

In case phase shifters are not used but switches, the optimization problem (12) shall be modified as follows

$$
\begin{aligned}
& \mathcal{P}_{\text {full-SW }}:[\mathbf{P}]_{m, n}=b_{m, n}, \\
& \mathcal{P}_{\text {interleaved-SW }}:[\mathbf{P}]_{m, n}=b_{m, n}\left[\mathbf{1}_{\kappa} \otimes \mathbf{I}_{N_{R F}}\right]_{m, n}, \\
& \mathcal{P}_{\text {localized-SW }}:[\mathbf{P}]_{m, n}=b_{m, n}\left[\mathbf{I}_{N_{R F}} \otimes \mathbf{1}_{\kappa}\right]_{m, n},
\end{aligned}
$$

where $b_{m, n} \in\{0,1\}$ and for $m=1, \ldots, Q \quad n=1, \ldots, N_{\mathrm{RF}}$.

\section{Alternating Analog-Digital Optimization}

In light of the previous section, it is evident that the analogdigital beamforming design is a coupled problem between the analog and the digital optimization. In the following, we propose an alternating optimization that iteratively computes the analog and digital part sequentially. While the digital part can be solved efficient by known methods, the analog part requires a novel technique.

\section{A. Hybrid Analog-Digital Beamforming}

It can be observed that the optimization problem (12) is coupled within the variables $\mathbf{w}$ and $\mathbf{P}$. In order to solve this obstacle, we consider an alternate iterative optimization. Precisely, in the $n$-th iteration, we have an optimal analog beamforming solution, $\mathbf{P}^{(n)}$, the corresponding digital beamforming can be obtained by solving

$$
\begin{aligned}
& \underset{\mathbf{w}^{(n+1)}}{\operatorname{maximize}}\left|\mathbf{h}^{H} \mathbf{P}^{(n)} \mathbf{w}^{(n+1)}\right|^{2} \\
& \text { subject to } \\
& \left|\mathbf{g}_{k}^{H} \mathbf{P}^{(n)} \mathbf{w}^{(n+1)}\right|^{2} \leq \epsilon_{k} \quad k=1, \ldots, K, \\
& \left|\left[\mathbf{P}^{(n)} \mathbf{w}^{(n+1)}\right]_{q}\right|^{2} \leq 1 / Q \quad q=1, \ldots, Q,
\end{aligned}
$$


The optimization problem in (20) can be cast as a SOCP as described in [6]. Once the optimal solution of (20) is obtained, $\mathbf{w}^{(n+1)}$, it is used for obtaining the analog beamforming design. As it is described in the previous section, the analog beamforming design depends on $\mathcal{P}$. Considering the case where the analog part is formed by phase shifters, given $\mathbf{w}^{(n)}$, the analog part can be obtained as follows

$$
\begin{aligned}
& \underset{\mathbf{p}^{(n+1)}}{\operatorname{maximize}}\left|\mathbf{h}^{H} \mathbf{W}^{(n)} \mathbf{p}^{(n+1)}\right|^{2} \\
& \text { subject to }\left|\mathbf{g}_{k}^{H} \mathbf{W}^{(n)} \mathbf{p}^{(n+1)}\right|^{2} \leq \epsilon_{k} \quad k=1, \ldots, K, \\
& \left|\left[\mathbf{W}^{(n)} \mathbf{p}^{(n+1)}\right]_{q}\right|^{2} \leq 1 / Q \quad q=1, \ldots, Q, \\
& \left|\left[\mathbf{p}^{(n+1)}\right]_{q}\right|^{2}=[\operatorname{vec}(\mathbf{C})]_{q} \quad q=1, \ldots, Q N_{\mathrm{RF}},
\end{aligned}
$$

where

$$
\begin{aligned}
& \mathbf{p}^{(n+1)}=\operatorname{vec}\left(\mathbf{P}^{(n+1), T}\right), \\
& \mathbf{W}^{(n)}=\mathbf{I}_{Q} \otimes \mathbf{W}^{(n+1), T} \in \mathbb{C}^{Q \times Q N_{\mathrm{RF}}} .
\end{aligned}
$$

Moreover, matrix $\mathbf{C}$ collapses the connectivity schemes such as

$\mathbf{C}_{\text {full }}=\mathbf{1}_{Q} \otimes \mathbf{1}_{N_{R F}}^{T}, \mathbf{C}_{\text {interleaved }}=\mathbf{1}_{\kappa} \otimes \mathbf{I}_{N_{R F}}, \mathbf{C}_{\text {localized }}=\mathbf{I}_{N_{R F}} \otimes \mathbf{1}_{\kappa}$

Whenever instead of phase shifters, switches are used, the optimization problem in (21) shall be modified as follows

$$
\begin{aligned}
& \underset{\mathbf{p}^{(n+1)}}{\operatorname{maximize}}\left|\mathbf{h}^{H} \mathbf{W}^{(n)} \mathbf{p}^{(n+1)}\right|^{2} \\
& \text { subject to }(21 \mathrm{~b}),(21 \mathrm{c}), \\
& {\left[\mathbf{p}^{(n+1)}\right]_{q}\left(\left[\mathbf{p}^{(n+1)}\right]_{q}-[\operatorname{vec}(\mathbf{C})]_{q}\right)=0 \quad q=1, \ldots, Q N_{\mathrm{RF}}}
\end{aligned}
$$

Remarkably, the constraint in (25c) imposes 0 or 1 to the analog component whether $[\operatorname{vec}(\mathbf{C})]_{q}=1$ and it imposes a 0 in case $[\operatorname{vec}(\mathbf{C})]_{q}=0$.

In both optimization problems (21) and (25) the optimization problem becomes a QCQP with equality constraints. The SDR followed by a randomization fails in providing a feasible point for these type of problems as discussed in [23], [27]. Let us remark this fact in the following.

Considering the SDP solution of either (21) or (25) relaxed problems $\left(\mathbf{P}^{*}\right)$ not being rank one, the Gaussian randomization technique computes a vector Gaussian random variable with zero mean and covariance matrix $\mathbf{P}^{*}$. With this variable, $\mathbf{p}^{\text {rand }}$, the system designer has to obtain a feasible solution of either (21) or (25). This feasible solution is imposed by the equality restrictions which require that the randomization is transformed into either a phase-only solution or a binary one. After this transformation, the interference and per-antenna power constraints are generally violated. Numerically, we observed the performance of SDR and Gaussian randomization for solving (21) and (25) and we validate that for 100 realizations and 1 interference, $10^{7}$ Gaussian randomizations are unable to yield to a feasible solution.

This fact differs to other optimizations that have used SDR where the scaling factor is optimized for not violating the constraints. This technique cannot be applied here as there is no flexibility in the scaling factor. As a result, the SDR leading to a high rank solution has an enormous difficulty of delivering a feasible rank one solution due to the equality constraints.

In order to solve the analog beamforming design with phase shifters, the authors in [1], [23] propose a non-smooth method based on [12] that behaves well. In the following, we propose a novel approach to solve both (21) and (25) that yield an efficient solution with an a priori substantially lower computational complexity. In the simulations Section, we compare the proposed analog beamforming optimization with [23]. In any case, we can anticipate that the presented approach is more computationally efficient since it performs at each iteration a SOCP in contrast to the work in [1] which requires to solve a sequence of semidefinite programs which are known to have a larger computational complexity [28].

\section{B. Penalty CCP for Analog Beamforming}

The underlying fact that makes the optimization problem (21) non-convex are the quadratic forms of both the objective function and the constraints (21d). Writing (21) in standard form, we obtain

$$
\begin{aligned}
& \underset{\mathbf{p}}{\operatorname{minimize}} \mathbf{p}^{H}(-\overline{\mathbf{H}}) \mathbf{p} \\
& \text { subject to } \\
& \mathbf{p}^{H} \overline{\mathbf{G}_{k}} \mathbf{p} \leq \epsilon_{k} \quad k=1, \ldots K, \\
& \left|[\mathbf{W} \mathbf{p}]_{q}\right|^{2} \leq 1 / Q \quad q=1, \ldots, Q \\
& \mathbf{p}^{H} \mathbf{E}_{i} \mathbf{p} \leq[\operatorname{vec}(\mathbf{C})]_{i} \quad i=1, \ldots, Q N_{R F}, \\
& \mathbf{p}^{H}\left(-\mathbf{E}_{i}\right) \mathbf{p} \leq[\operatorname{vec}(\mathbf{C})]_{i} \quad i=1, \ldots, Q N_{R F} .
\end{aligned}
$$

where $\mathbf{E}_{i}$ is a $Q N_{R F} \times Q N_{R F}$ matrix whose entries are zero apart from the $i$-th diagonal entry which is equal to one.

$$
\begin{aligned}
\overline{\mathbf{H}} & =\mathbf{W}^{H} \mathbf{h} \mathbf{h}^{H} \mathbf{W}, \\
\overline{\mathbf{G}_{k}} & =\mathbf{W}^{H} \mathbf{g}_{k} \mathbf{g}_{k}^{H} \mathbf{W} .
\end{aligned}
$$

For the sake of simplicity, we have omitted the superscripts $(n)$ in all cases. By observing (26) it is indicated that the non-convexity of the problem is due to the objective function and the last set of constraints which are negative definite. In order to solve (26), we can apply penalty CCP [22]. The idea of this technique is to linearize the aforementioned nonconvex parts of the optimization problem by its linear convex approximation.

For any $\mathbf{p}, \mathbf{z} \in \mathbb{C}^{Q N_{R F} \times 1}$, the inequality

$$
(\mathbf{p}-\mathbf{z})^{H} \mathbf{X}(\mathbf{p}-\mathbf{z}) \leq 0
$$

where $\mathbf{X} \in \mathbb{C}^{Q N_{R F} \times Q N_{R F}}$ is negative definite can be expanded by

$$
\mathbf{p}^{H} \mathbf{X} \mathbf{p} \leq 2 \mathcal{R}\left\{\mathbf{z}^{H} \mathbf{X} \mathbf{p}\right\}-\mathbf{z}^{H} \mathbf{X} \mathbf{z}
$$

Therefore, using the linear restriction around the point $\mathbf{z}$, we 
might replace the non-convex inequalities in (26) so that

$$
\begin{aligned}
& \underset{\mathbf{p}}{\operatorname{maximize}} 2 \operatorname{Re}\left(\mathbf{z}^{H} \overline{\mathbf{H}} \mathbf{p}\right)-\mathbf{z}^{H} \overline{\mathbf{H}} \mathbf{z} \\
& \text { subject to } \\
& \mathbf{p}^{H} \overline{\mathbf{G}_{k}} \mathbf{p} \leq \epsilon_{k} \quad k=1, \ldots K, \\
& \left|[\mathbf{W} \mathbf{p}]_{q}\right|^{2} \leq 1 / Q \quad q=1, \ldots, Q \\
& \mathbf{p}^{H} \mathbf{E}_{i} \mathbf{p} \leq[\operatorname{vec}(\mathbf{C})]_{i} \quad i=1, \ldots, Q N_{R F}, \\
& \mathbf{z}^{H} \mathbf{E}_{i} \mathbf{z} \leq[\operatorname{vec}(\mathbf{C})]_{i}+2 \mathcal{R}\left\{\mathbf{z}^{H} \mathbf{E}_{i} \mathbf{p}\right\} \quad i=1, \ldots, Q N_{R F} .
\end{aligned}
$$

The optimization problem (31) is a SOCP that can be efficiently solved via gradient based methods. Under this context, given an initial feasible value of $\mathbf{z}, \mathbf{z}^{(0)}$, iteratively solving (31) leads to a Karush-Kuhn-Tucker (KKT) point of (26) [29].

Obtaining an initial feasible point $\mathbf{z}^{(0)}$ is as challenging as optimizing (26). In order to solve this problem, the authors in [22] impose the use of slack variables over all constraints and penalizing the objective function with its sum. With this, the optimization problem at the $t$-th iteration can be described as

$$
\underset{\mathbf{p}^{(t)}}{\operatorname{maximize}} 2 \mathcal{R}\left\{\mathbf{z}^{(t), H} \overline{\mathbf{H}} \mathbf{p}^{(t)}\right\}-\mathbf{z}^{(t), H} \overline{\mathbf{H}} \mathbf{z}^{(t)}-\beta^{(t)} \sum_{m=1}^{K+2 Q+M}
$$

subject to

$$
\mathbf{p}^{(t), H} \overline{\mathbf{G}_{k}} \mathbf{p}^{(t)} \leq \epsilon_{k}+s_{k} \quad k=1, \ldots K,
$$$$
\left|\left[\mathbf{W} \mathbf{p}^{(t)}\right]_{q}\right|^{2} \leq 1 / Q+s_{K+q} \quad q=1, \ldots, Q
$$$$
\mathbf{p}^{(t), H} \mathbf{E}_{i} \mathbf{p}^{(t)} \leq[\operatorname{vec}(\mathbf{C})]_{i}+s_{i+Q+K} \quad i=1, \ldots, M,
$$$$
\mathbf{z}^{(t), H} \mathbf{E}_{i} \mathbf{z}^{(t)} \leq[\operatorname{vec}(\mathbf{C})]_{i}+2 \mathcal{R}\left\{\mathbf{z}^{(t), H} \mathbf{E}_{i} \mathbf{p}^{(t)}\right\}+s_{i+K+Q+M}
$$$$
i=1, \ldots, M
$$$$
s_{m} \geq 0 \quad m=1, \ldots, K+2 Q+M, .
$$

where $\beta^{(t)}$ is a regularization factor that controls the feasibility of the constraints. For high values of $\beta$, the optimization focuses on yielding a feasible point for (31). On the other hand, for low values of $\beta$, the optimization problem targets to maximize the array gain towards the secondary user. This regularization factor can be updated over the iterations. For our case, we consider an additive update by a factor $\rho$. The algorithm is summarized in Algorithm 1.

As it can be observed, the proposed algorithm includes the stopping criteria $\left|\sum_{m=1}^{K+2 M+1} s_{m}\right| \leq \chi$. This condition guarantees that all the constraints of the original problem (26) are not violated for a sufficiently low $\chi$. Note that, it is possible to allow for different maximum violations of each constraint by weighting the penalty function $\sum_{m=1}^{K+2 M+Q} s_{m}$. In addition, the convergence of the solution is controlled by the condition $\left\|\mathbf{p}^{(t)}-\mathbf{p}^{(t-1)}\right\| \leq \nu$.

The role of $\beta$ is to balance the optimization of the array gain to the intended users and the minimization of the constraint violation (i.e for very high $\beta$ the optimization problem seeks for a feasible point rather than optimizing the array gain). We variate the value of $\beta$ over the different iterations. First, we set a relatively low value of $\beta^{(0)}$, and; posteriorly, we sequentially increase this value. In other words, the proposed scheme first focuses on maximizing the array gain to the secondary user and, later, it seeks for a feasible solution. To avoid $\beta$ taking a

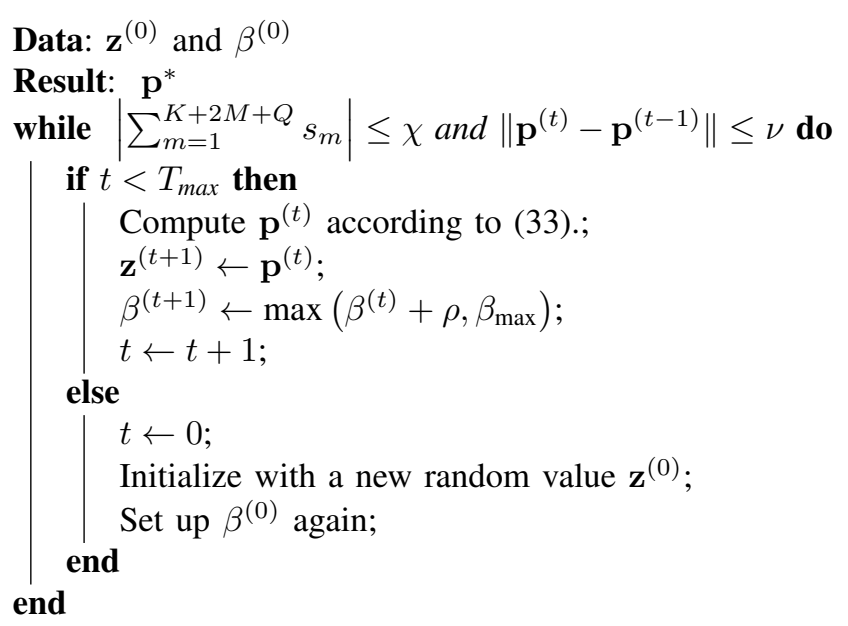

Output the final solution;

Algorithm 1: Penalty CCP optimization for analog beamforming optimization.

very large value when the number of iterations becomes large, slemading to numerical difficulties, we consider a maximum $\beta$ value $\beta_{\max }$.

Algorithm 1 does not guarantee convergence to a feasible problem in (26), as [22] mentions. This is controlled by imposing a maximum number of iterations $T_{\max }$ and, in case it is reached, we start with a new initial point.

Similarly to this optimization problem, we can convexify the non-convex constraints in (25) in order to apply the penalty CCP method as follows

$\underset{\mathbf{p}}{\operatorname{maximize}} 2 \mathcal{R}\left\{\mathbf{z}^{(t), H} \overline{\mathbf{H}} \mathbf{p}^{(t)}\right\}-\mathbf{z}^{(t), H} \overline{\mathbf{H}} \mathbf{z}^{(t)}-\beta \sum_{m=1}^{K+2 Q+M} s_{m}$

subject to

$\mathbf{p}^{(t), H} \overline{\mathbf{G}_{k}} \mathbf{p}^{(t)} \leq \epsilon_{l}+s_{k} \quad k=1, \ldots K$,

$\left|\left[\mathbf{W} \mathbf{p}^{(t)}\right]_{q}\right|^{2} \leq 1 / Q+s_{K+q} \quad q=1, \ldots, Q$

$\mathbf{p}^{(t), H} \mathbf{E}_{i} \mathbf{p}^{(t)}-\operatorname{vec}(\mathbf{C})^{T} \mathbf{E}_{i} \mathbf{p}^{(t)} \leq+s_{i+Q+K} \quad i=1, \ldots, M$,

$\mathbf{z}^{(t), H} \mathbf{E}_{i} \mathbf{z}^{(t)}-\operatorname{vec}(\mathbf{C})^{T} \mathbf{E}_{i} \mathbf{p}^{(t)} \leq+2 \mathcal{R}\left\{\mathbf{z}^{(t), H} \mathbf{E}_{i} \mathbf{p}^{(t)}\right\}+s_{i+K+Q+M}$

$i=1, \ldots, M$,

$s_{m} \geq 0 \quad m=1, \ldots, K+2 Q+M$.

Under this context, Algorithm 1 can be applied. For this case, we can obtain a solution $\mathbf{p} \in\{0,1\}$ that it does not violate the constraints.

\section{Alternating Optimization}

In the previous subsection, penalty $\mathrm{CCP}$ method for dealing with the analog processing optimization is presented. Bearing this mechanism in mind, it is proposed an alternating optimization which sequentially obtains efficient solutions of the analog and digital parts. The method is depicted in Algorithm 2.

As general non-convex approximation techniques, the performance of the method relies on how close the initial value, $\mathbf{P}^{(0)}$, is to the optimal value $\mathbf{P}^{*}$. We use the following initial 
Data: $\mathbf{P}^{(0)}$ considering the sub-array connectivity $\mathbf{C}$

Result: $\mathbf{w}^{*}$ and $\mathbf{P}^{*}$

initialization ;

while $\left|\mathbf{P}^{(n)} \mathbf{w}(n)-\mathbf{P}^{(n+1)} \mathbf{w}^{(n+1)}\right|^{2} \leq \psi$ do

Compute $\mathbf{w}^{(n+1)}$ considering $\mathbf{P}^{(n)}$, ;

Compute $\mathbf{P}^{(n+1)}$ with the penalty CCP method considering $\mathbf{w}^{(n+1)}$;

$n \leftarrow n+1$;

end

Output the final solution;

Algorithm 2: Alternate analog-digital optimization.

value

$$
\mathbf{P}^{(0)}=\left(\mathbf{v}_{\text {analog }} \otimes \mathbf{1}_{N_{R F}}^{T}\right) \circ \mathbf{C},
$$

where $\mathbf{v}_{\text {analog }}$ is the solution obtained of the following optimization problem

$$
\begin{aligned}
& \underset{\mathbf{v}}{\operatorname{maximize}}\left|\mathbf{h}^{H} \mathbf{v}\right|^{2} \\
& \text { subject to } \\
& \left|\mathbf{h}_{i_{k}}^{H} \mathbf{v}\right|^{2} \leq \epsilon_{k} \quad k=1, \ldots, K, \\
& \left|[\mathbf{v}]_{i}\right|^{2}=1 / Q \quad i=1, \ldots, Q .
\end{aligned}
$$

As reported in [23] this optimization problem can be efficiently solved via non-smooth methods. The assumed heuristic is that the phase-only analog beamforming solution is close to the hybrid analog beamforming case, despite not all the antennas are used and independent of the analog component assumed (phase shifter or switch).

Remarkably, little is known regarding the convergence of non-convex alternating optimization problems from the theoretical point of view. Indeed, there is no theoretical evidence that Algorithm 2 converges. Fortunately, over all the different realizations we perform in the numerical evaluation we observe that the alternating optimization converges more than $80 \%$ of the realizations. Prior to the numerical validation of our approach, we evaluate its theoretical computational complexity.

\section{Computational Complexity Analysis}

The proposed alternating projection method consists of a set of sequential SOCP. Bearing in mind that the worst-case asymptotic complexity (WCAC) of the SOCP is $\mathcal{O}\left((n+L)^{3.5}\right)$, where $n$ is variable dimension and $L$ the number of inequalities [30], the digital beamforming presents a WCAC of

$$
\mathrm{WCAC}_{\text {digital }}=\mathcal{O}\left(\left(4 Q+2 K+4+2 N_{R F}\right)^{3.5}\right) .
$$

Similarly, the WCAC of the analog optimization becomes

$$
\begin{aligned}
& \mathrm{WCAC}_{\text {analog-PS }}=\mathcal{O}\left(I_{\text {PCCP-PS }}\left(12 Q N_{R F}+4 K\right)^{3.5}\right), \\
& \mathrm{WCAC}_{\text {analog-SW }}=\mathcal{O}\left(I_{\text {PCCP-SW }}\left(6 Q N_{R F}+2 K\right)^{3.5}\right),
\end{aligned}
$$

for the case we employ phase shifters or switches. $I_{\text {PCCP-PS }}, I_{\text {PCCP-SW }}$ denote the average worst-case number of iterations required by the penalty $\mathrm{CCP}$ method to converge.
Note that the WCAC is independent of the elected connectivity as the number of constraints remain the same.

In light of the above discussion and based on the composition rule of the asymptotic analysis, the WCAC is dominated by the analog processing scheme so that the overall WCAC becomes

$\mathrm{WCAC}_{\text {Alternating-PS }}=\mathcal{O}\left(I_{\text {Alternating }} I_{\text {PCCP-PS }}\left(12 Q N_{R F}+4 K\right)^{3.5}\right)$,

in case phase shifters are used and

$\mathrm{WCAC}_{\text {Alternating-SW }}=\mathcal{O}\left(I_{\text {Alternating }} I_{\mathrm{PCCP}-\mathrm{SW}}\left(6 Q N_{R F}+2 K\right)^{3.5}\right)$,

for the switches case. The variable $I_{\text {Alternating }}$ denotes the worstcase number of required iterations for solving the alternating optimization.

\section{NUMERICAL RESULTS}

This section presents the numerical validation of the proposed method. The beamforming schemes are evaluated over 500 realizations considering the channel model described in Section II. In all cases, we present the empirical cumulative distribution function (CDF) of the secondary user spectral efficiency

$$
\text { Spectral Efficiency }=\log _{2}\left(1+\left|\mathbf{h}^{H} \mathbf{P} \mathbf{w}\right|^{2}\right) .
$$

As a benchmark, we consider the achievable rates obtained via digital beamforming when optimizing (11). We consider both $\mathbf{H}$ and $\left\{\mathbf{G}_{k}\right\}_{k=1}^{K}$ are distributed according to the Section II described channel model. Since in the overall paper we consider a backhauling scenario, we set $L=5$ and $C=1$ according to the 'above the roof top' channel model presented in [25].

According to [25], in a backhauling scenario the small scale fading can be modelled as

$$
\alpha_{c l}=A_{c l} e^{\psi_{c l} j},
$$

where $A_{c l}$ is Rayleigh distributed with mean 0.1 and $\psi_{c l}$ is uniformly distributed from 0 to $2 \pi$. We assume that $\theta_{11}^{t x}, \theta_{11}^{r x}$ are deterministic and it can be computed by known the relative positions between the transmitter and the receiver. On the other hand, for $c>1$ and $l>1$, we assume that

$$
\begin{aligned}
& \theta_{c l}^{t x}=\theta_{11}^{t x}+\chi^{t x}, \\
& \theta_{c l}^{r x}=\theta_{11}^{r x}+\chi^{r x},
\end{aligned}
$$

where $\chi^{t x}$ and $\chi^{r x}$ are zero mean Gaussian distributed random variables with standard deviation equal to 5 .

We consider that $\gamma_{s}=\gamma_{p, k}=P=1$ for $k=1, \ldots, K$. In addition, the fixed receiver beamformers of the secondary and primary users are considered to be the matched filter to the direction of arrival of the deterministic ray.

For the sake of simplicity in this first evaluation we assume only one interference $(K=1)$ that requires to be minimized $\epsilon=-30 \mathrm{~d} B$. In a preliminary analysis we consider the comparison of the analog beamforming with phase shifter non-smooth optimization in [23] and the PCCP method that solves (21) as described in Algorithm 1. Figures 4 and 5 
show the average array gain $\left(20 \log \left(\left|\mathbf{h}^{H} \mathbf{v}\right|\right)\right)$ and the average processing time. It has been assumed a fully-connected array with different number of antennas and the simulations have been performed in a with a Windows desktop with 4 Intel i5 cores and 4GB of RAM.

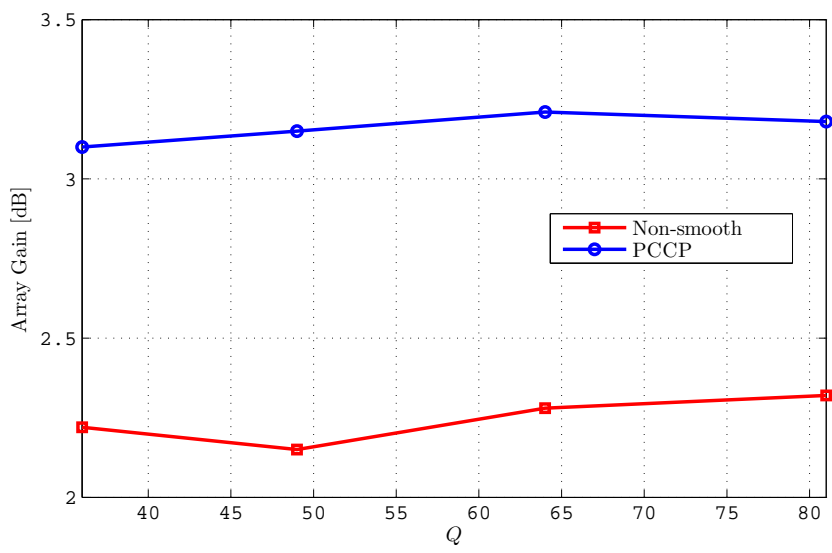

Fig. 4: Array gain $\left(20 \log \left(\left|\mathbf{h}^{H} \mathbf{v}\right|\right)\right)$ comparison of the nonsmooth method versus the proposed PCCP.

It can be observed in Figure 4 that PCCP behaves slightly better than the benchmark non-smooth scheme (i.e. the array gain difference is less than $1 \mathrm{~dB}$ ). On the other hand, as predicted in previous sections, the computational time severely increases with the number of antennas. For instance, in the 81 antenna case, the non-smooth method presents 6 times higher processing time compared to the PCCP.

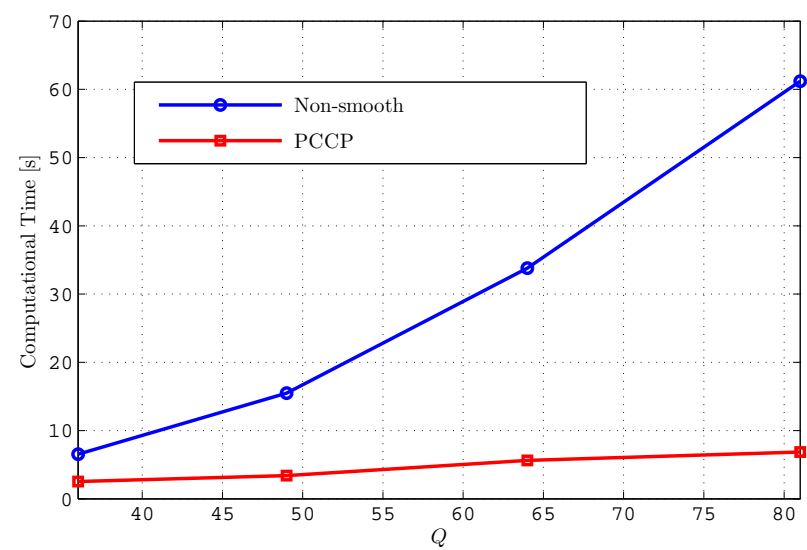

Fig. 5: Average processing time of the analog beamforming optimization of the non-smooth and the PCCP.

This numerical result motivates the use of PCCP in the considered scenario as for mmWave communications, large number of antenna arrays are expected. In the following alternating optimization performance results, we omit the comparison with the non-smooth method in the phase shifter analog component case as similar behaviour is expected in most of the cases due to computational complexity difference of each scheme.
The alternating optimization parameters in Algorithm 2 are set so that

$$
\psi=10^{-1}, \chi=\nu=10^{-2}, T_{\max }=50, \beta^{(0)}=10, \rho=10 .
$$

Considering this setting, there are a plethora of possible evaluations which cannot be presented in here due to space limitations. In the following we present the most relevant results. It is important to remark that for each realization we perform 5 trials and we take the solution which yields to the highest array gain.

We first show an example of the beamforming design for an array of $Q=8$ and $N_{R F}=2$. Figure 6 shows the array gain when the AoD of the intended user is located at 0 degrees and the interference is at 30 degrees. In the Figure is also depicted the different scatters rays obtained in the realization. It can be observed that the digital design is the one offering the largest array gain. For this case, it is also observed that the localized scheme with phase shifters can attain an array gain very close to the optimal fully-digital design. In all cases it can be checked that the interference power level is bellow $30 \mathrm{dBs}$.

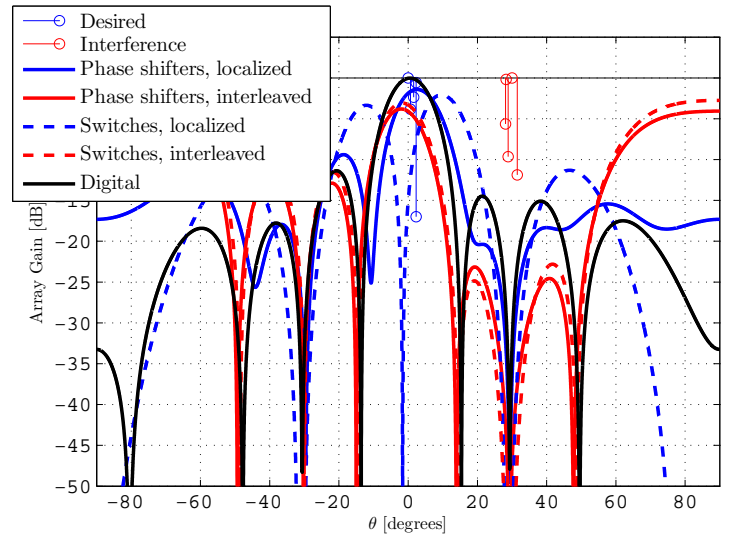

Fig. 6: Array gain $\left(20 \log \left(\left|\mathbf{h}^{H} \mathbf{P} \mathbf{w}\right|\right)\right)$ example with $Q=8$, $N_{R F}=2$. The AoD of the desired user is 0 degrees while the interference $\mathrm{AoD}$ is located at 30 degrees.

Before evaluating the spectral efficiency we investigate the efficiency of Algorithm 2. Figure 7 shows the average number of alternating iterations required to reach a solution for either phase shifters or switches considering all types of connectivity matrices and $Q=8,16$ and 32. We have also assumed $N_{R F}=$ 2. In all cases, the average number of iterations is below 5.5. This numerical result supports the efficiency of the conceived technique which is shown to quickly reach a solution. It is important to remark that phase shifters require more iterations than switches.

Figures 8 depicts the achievable data rates when using phase shifters and different connectivity matrices considering $Q=16$ and $N_{R F}=2,4,6$. We have omitted the fullyconnected case as we know from [8] that the hybrid ana$\log$ digital beamforming with phase shifters can attain the same values as for the digital beamforming design. It is observed that whenever partial connections are considered, lower achievable are obtained. In addition, having $N_{R F} \geq 2$ 


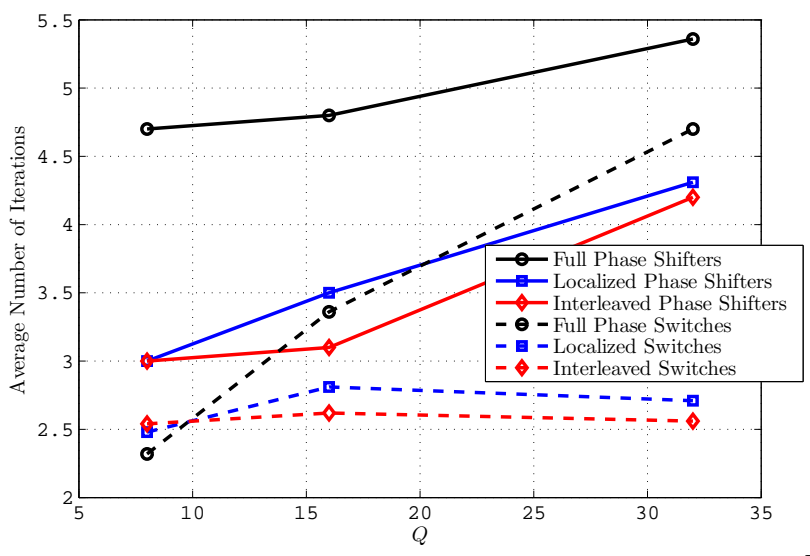

Fig. 7: Average number of iterations for different $Q$, connectivity matrices for either phase shifters or switches.

favors the performance of the systems. This differs from the fully-connected case, where it is known that $N_{R F}=2$ can achieve a data rate as large as the digital case. As the Figure describes, the localized connectivity matrix shows a slightly better data rate compared to the interleaved case.

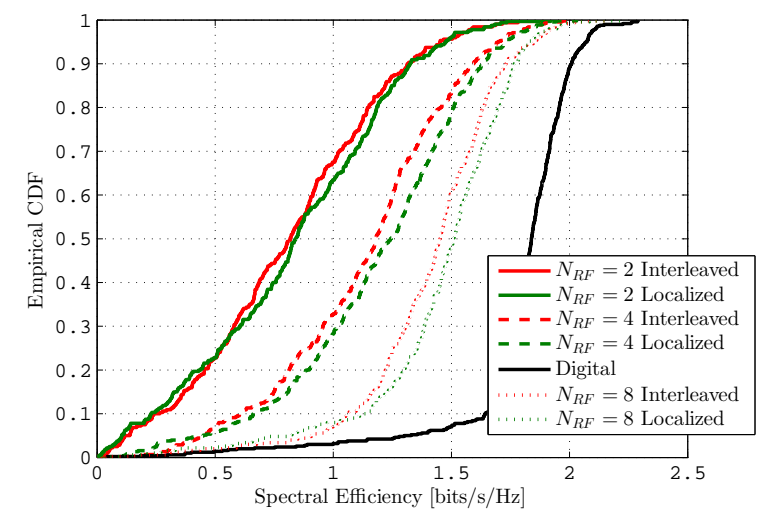

Fig. 8: Empirical CDF of the achievable data rates for different $N_{R F}$ and connectivity matrices with phase shifters analog components.

For the sake of completeness, let us compare the performance of the proposed method in the fully-connected with phase shifters with respect to the fully-digital alternative. The aim of this comparison is to show how the proposed method is able to behave closely to its upper-bound. This is shown in Figure 9 for $N_{R F}=2$ and $Q=8,16,32$ and 64 . It can be observed that the conceived alternating optimization results in a performance degradation below to $1.5 \mathrm{~dB}$ with respect to the upper bound. In other words, the performance loss of employing the proposed convex relaxation approach appears to be low.

As described in previous sections, other mmWave optimization methods based on the Frobenius norm minimization might fail in providing an efficient solution to the considered problem. Considering [26] as benchmark for the fully-connected architecture with phase shifters since it is a method that takes

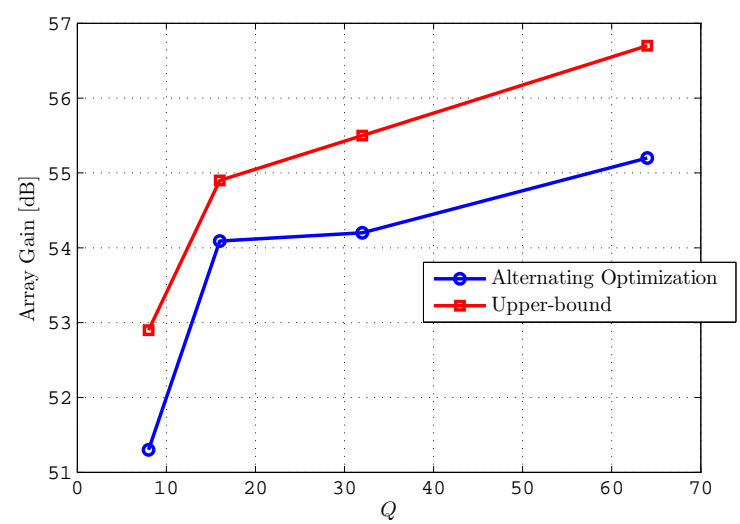

Fig. 9: Average array gain upper-bound comparison for different $Q$ values and $N_{R F}=2$

into account per-antenna power constraints, we evaluate the interference power limit defined as

$$
\mathcal{I} \mathcal{T}_{k}=20 \log \left(\left|\mathbf{g}_{k}^{H} \mathbf{P}_{\text {benchmark }} \mathbf{w}_{\text {benchmark }}\right|\right),
$$

where $\mathbf{P}_{\text {benchmark }}$ and $\mathbf{w}_{\text {benchmark }}$ are obtained via the proposed method in [26] considering the fully-digital solution (i.e. the optimal solution of (13)). The results in Figure 10 are obtained for $N_{R F}=2, Q=8,16,32$ and 64 ; and a maximum power constraint of $\epsilon=-30 \mathrm{~dB}$. It is evident that in all cases, the beamforming solution exceeds the maximum interference power level. Bearing this in mind, the alternative in [26] does not offer a valid design since the interference power limits imposed by the spectrum sharing scenario are violated. On the other hand, our contribution always delivers a feasible solution with $\mathcal{I} \mathcal{T}$ below the imposed $\epsilon$ constraint. Note that we have assumed $K=1$.

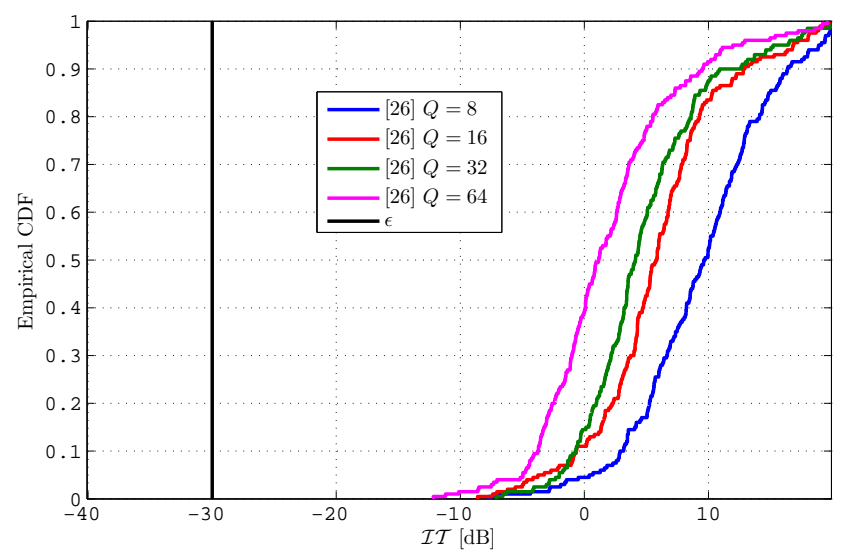

Fig. 10: Interference power limit for different $Q$ given $N_{R F}=$ 2. It can be observed that the method in [26] violates the interference power constraint in all realizations.

Furthermore, we consider the comparison of the proposed method with [16], [20]. Note that neither [16] nor [20] assume per-antenna power constraints as this paper considers. In order to provide a fair comparison, we compute a sub-optimal 


\begin{tabular}{|l|l|l|l|}
\hline$Q$ & $K=1$ & $K=2$ & $K=3$ \\
\hline 8 & $24.5 \%$ & $88 \%$ & $94 \%$ \\
\hline 16 & $23.2 \%$ & $56 \%$ & $63 \%$ \\
\hline 32 & $19 \%$ & $40 \%$ & $46 \%$ \\
\hline 64 & $13 \%$ & $34 \%$ & $39 \%$ \\
\hline
\end{tabular}

TABLE I: Percentage of interference constraints violation with the PE-AltMin technique in [20].

solution of the mechanisms described in [16], [20] by rescaling the resulting precoding solution with a factor in order to meet the per-antenna power constraints.

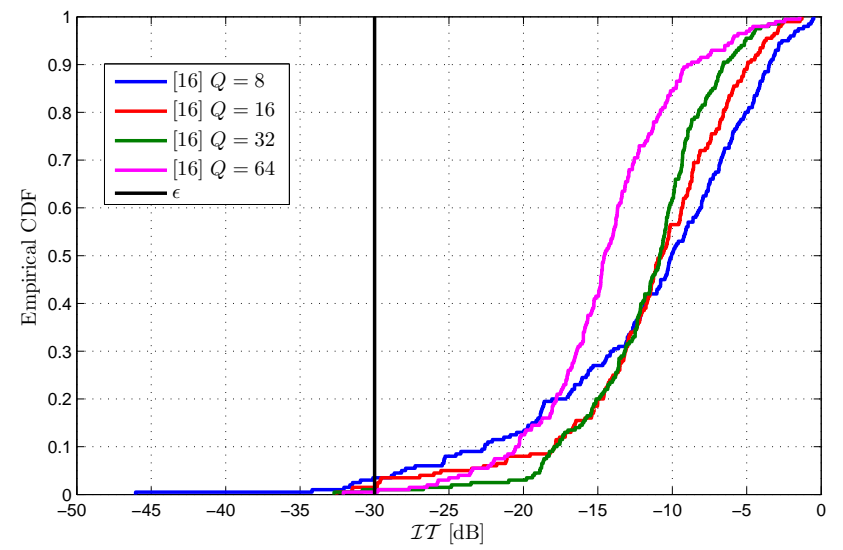

Fig. 11: Interference power limit for different $Q$ given $N_{R F}=$ 2 . It can be observed that the method in [16] violates the interference power constraint in all realizations.

The same setting as for computing the results in Figure 10 has been employed in Figure 11. Again, it can be observed that in all cases the obtained solution exceeds the power interference limit for the considered $Q$ values.

The results of PE-AltMin algorithm from [20] are depicted in Table I where it is shown the interference constraints violation percentage during the Monte Carlo runs. That is, whenever

$$
\mathcal{I T}_{k}>\epsilon, \quad \forall k
$$

This is done for $K=1,2$ and 3 and it is presented in $\%$. As it can be observed, PE-AltMin provides low percentage of interference violation when $K=1$. Nevertheless, when $K$ increases, the number of interference power limits constraints violation increases, leading to unacceptable values even for large $Q$. This result motivates the proposed design which considers the interference constraints in the optimization process and; thus, it ensures its inviolability.

Continuing with the spectral efficiency evaluation, we consider switches as analog component in Figure 12. For this case we observe a clear performance gain when fully-connected is considered compared to the partial connected designs. Again, the larger $N_{R F}$ the larger achievable rates are obtained. In contrast to when phase shifters as analog components are used,

\begin{tabular}{|l|l|l|}
\hline Connectivity \& Analog Components & Phase-shifters [bits/s/Hz] & Switches [bits/s/Hz] \\
\hline Interleaved & 0.8 & 0.12 \\
\hline Localized & 0.8 & 0.12 \\
\hline Full & 1.76 & 0.3 \\
\hline
\end{tabular}

TABLE II: Average achievable data rates for $N_{R F}=2$ different connectivity matrices and analog components.

\begin{tabular}{|l|l|l|}
\hline Connectivity \& Analog Components & Phase-shifters [bits/s/Hz] & Switches [bits/s/Hz] \\
\hline Interleaved & 1.18 & 0.5 \\
\hline Localized & 1.13 & 0.51 \\
\hline Full & 1.76 & 0.74 \\
\hline
\end{tabular}

TABLE III: Average achievable data rates for $N_{R F}=4$ different connectivity matrices and analog components.

in Figure 12 we do not observe any relevant different between interleaved and localized connectivity schemes.

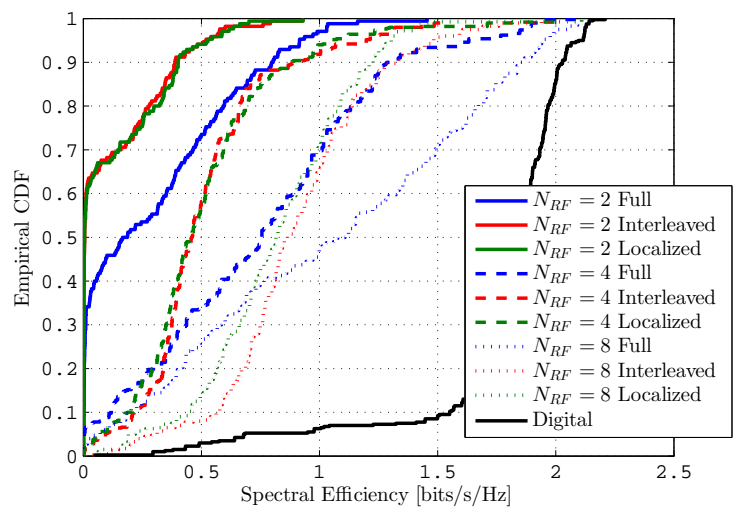

Fig. 12: Empirical CDF of the achievable data rate for different $N_{R F}$ and connectivity matrices with switches analog components.

Tables II, III and IV depict the average data rates for each of considered techniques described in previous Figures. It can be observed that in all connectivity matrices and $N_{R F}$ values the phase shifters analog component is the one that offers larger data rates compared to the switches case. As the RF chains are increased, the performance of the hybrid analog-digital with partial connectivity designs becomes closer to the fully-digital case. Precisely, with $N_{R F}=8$, the hybrid solution with phase shifter and interleaved connectivity loses a $17 \%$ of the spectral efficiency.

The differences between interleaved and localized schemes are minimal for all cases. Moreover, the use of fully-connected $\mathrm{BN}$ always increases the data rates, especially when phase shifters are used.

In order to appreciate the effect of increasing the number of antennas we compare two cases with the same number of BN connections. This is, we consider the former $Q=16, N_{R F}=$

\begin{tabular}{|l|l|l|}
\hline Connectivity \& Analog Components & Phase-shifters [bits/s/Hz] & Switches [bits/s/Hz] \\
\hline Interleaved & 1.46 & 0.9 \\
\hline Localized & 1.41 & 0.81 \\
\hline Full & 1.76 & 1.04 \\
\hline
\end{tabular}

TABLE IV: Average achievable data rates for $N_{R F}=8$ different connectivity matrices and analog components. 
4 and we compare the achievable data rates of a scheme with $Q=32, N_{R F}=2$. As the Figures 13 and 14 present, whenever there is an increase on the RF chains, despite we decrease the number of antennas, the resulting beamformer design yields to higher data rates. This effect is notorious when switches are considered (Figure 14). On the other hand, the difference with phase shifters are used is low as reported in Figure 13.

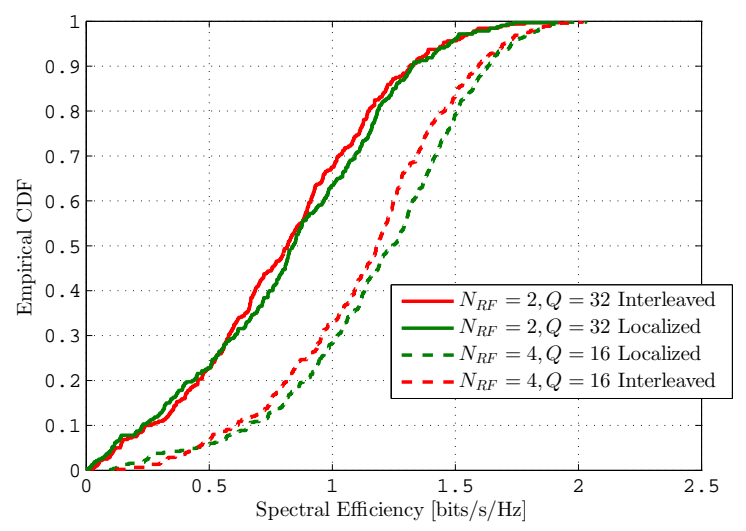

Fig. 13: Empirical CDF of the achievable data rate for different $Q, N_{R F}$ and connectivity matrices with phase shifters analog components.

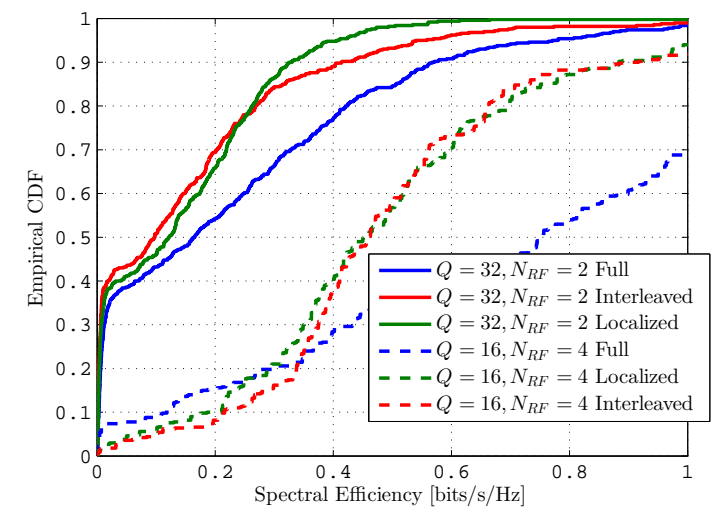

Fig. 14: Empirical CDF of the achievable data rate for different $Q, N_{R F}$ and connectivity matrices with switches analog components.

Finally we show the performance when varying $K$ and $\epsilon$ in Figure 15 . We consider a setting with a fully-connected array with phase-shifters having $Q=64, N_{R F}=2$ and a transmit power of $10^{4}$ Watts. It is evident that by increasing $K$ and decreasing $\epsilon$ the array gain is reduced. The reduction is more notorious for the $K=3, \epsilon=-30 d B$ case.

\section{CONCLUSIONS}

This work presents an optimization framework for obtaining efficient hybrid analog-digital transmit beamforming designs for spectrum sharing scenarios. The joint optimization of both, the analog and digital parts, is solved via an alternating optimization method that sequentially optimizes the analog and

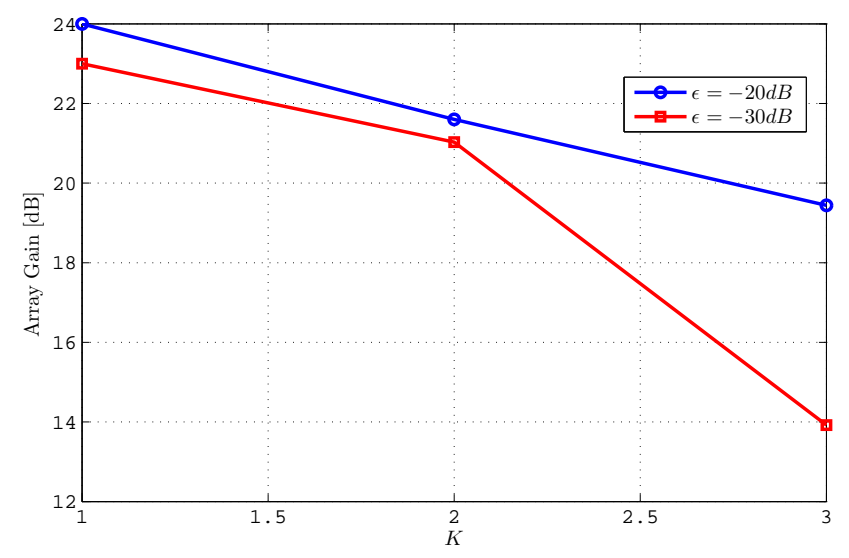

Fig. 15: Average array gain for different values of $K$ and $\epsilon$.

digital parts. While the digital part can be cast as a SOCP, the analog part is non-convex optimization problem that requires a novel optimization tool. It is shown that the conceived penalty CCP for the analog optimization behaves well for both the phase shifters and the switches case. The numerical results show the performance comparison of all tentative analog components under ideal conditions (no losses and no hardware impairments). These results are of great importance in next generation spectrum sharing backhaul networks design as they describe the best election in terms of ideal performance for realizable hybrid analog-digital beamforming architectures.

It is important to remark that the presented contribution tackles two unexplored fields on the mmWave precoding subject: the incorporation of spatial constraints and the use of switches. These two aspects force us two resort to methods that completely differ to existing alternatives based on the minimization of the Frobenius norm of the hybrid analogdigital solution with respect to the fully-digital design. Indeed, we showed that the minimization of the Frobenius norm suffers from a large performance degradation since the interference constraints are violated. In addition, the PCCP method for the beamforming optimization with switches and phase shifters is novel and in the paper we have shown its great potential as it is able to deliver efficient solutions with a low computational complexity even for large beamforming networks.

\section{REFERENCES}

[1] M. A. Vázquez, L. Blanco, X. Artiga, and A. Pérez-Neira, "Hybrid analog-digital transmit beamforming for spectrum sharing satelliteterrestrial systems," in 2016 IEEE 17th International Workshop on Signal Processing Advances in Wireless Communications (SPAWC), July 2016, pp. 1-8.

[2] U. Siddique, H. Tabassum, E. Hossain, and D. I. Kim, "Wireless backhauling of $5 \mathrm{G}$ small cells: challenges and solution approaches," IEEE Wireless Communications, vol. 22, no. 5, pp. 22-31, October 2015.

[3] A. K. Gupta, J. G. Andrews, and R. W. Heath, "On the Feasibility of Sharing Spectrum Licenses in mmWave Cellular Systems," IEEE Transactions on Communications, vol. 64, no. 9, pp. 3981-3995, Sept 2016.

[4] H. Shokri-Ghadikolaei, F. Boccardi, C. Fischione, G. Fodor, and M. Zorzi, "Spectrum Sharing in mmWave Cellular Networks via Cell Association, Coordination, and Beamforming," IEEE Journal on Selected Areas in Communications, vol. PP, no. 99, pp. 1-1, 2016. 
[5] A. K. Gupta, A. Alkhateeb, J. G. Andrews, and R. W. Heath, "Gains of Restricted Secondary Licensing in Millimeter Wave Cellular Systems," IEEE Journal on Selected Areas in Communications, vol. 34, no. 11, pp. 2935-2950, Nov 2016.

[6] R. Zhang and Y. C. Liang, "Exploiting Multi-Antennas for Opportunistic Spectrum Sharing in Cognitive Radio Networks," IEEE Journal of Selected Topics in Signal Processing, vol. 2, no. 1, pp. 88-102, Feb 2008.

[7] J. A. Zhang, X. Huang, V. Dyadyuk, and Y. J. Guo, "Massive hybrid antenna array for millimeter-wave cellular communications," IEEE Wireless Communications, vol. 22, no. 1, pp. 79-87, February 2015.

[8] F. Sohrabi and W. Yu, "Hybrid Digital and Analog Beamforming Design for Large-Scale Antenna Arrays," IEEE Journal of Selected Topics in Signal Processing, vol. 10, no. 3, pp. 501-513, April 2016.

[9] L. Liang, W. Xu, and X. Dong, "Low-Complexity Hybrid Precoding in Massive Multiuser MIMO Systems," IEEE Wireless Communications Letters, vol. 3, no. 6, pp. 653-656, Dec 2014.

[10] J. Geng, Z. Wei, X. Wang, W. Xiang, and D. Yang, "Multiuser hybrid analog/digital beamforming for relatively large-scale antenna arrays," in Globecom Workshops (GC Wkshps), 2013 IEEE, Dec 2013, pp. 123128.

[11] T. E. Bogale and L. B. Le, "Beamforming for multiuser massive MIMO systems: Digital versus hybrid analog-digital," in Global Communications Conference (GLOBECOM), 2014 IEEE, Dec 2014, pp. 4066-4071.

[12] A. H. Phan, H. D. Tuan, H. H. Kha, and D. T. Ngo, "Nonsmooth Optimization for Efficient Beamforming in Cognitive Radio Multicast Transmission," IEEE Transactions on Signal Processing, vol. 60, no. 6, pp. 2941-2951, June 2012.

[13] C. G. Tsinos, S. Maleki, S. Chatzinotas, and B. Ottersten, "Hybrid analog-digital transceiver designs for cognitive radio millimiter wave systems," in 2016 50th Asilomar Conference on Signals, Systems and Computers, Nov 2016, pp. 1785-1789.

[14] R. Haupt, "Null synthesis with phase and amplitude controls at the subarray outputs," IEEE Transactions on Antennas and Propagation, vol. 33, no. 5, pp. 505-509, May 1985.

[15] A. A. Khan and A. K. Brown, "Sector nulling in planar irregular subarrayed sparse array antennas," IET Microwaves, Antennas Propagation, vol. 10, no. 1, pp. 25-30, 2016.

[16] O. E. Ayach, S. Rajagopal, S. Abu-Surra, Z. Pi, and R. W. Heath, "Spatially Sparse Precoding in Millimeter Wave MIMO Systems," IEEE Transactions on Wireless Communications, vol. 13, no. 3, pp. 14991513, March 2014.

[17] H. Ghauch, T. Kim, M. Bengtsson, and M. Skoglund, "Subspace Estimation and Decomposition for Large Millimeter-Wave MIMO Systems," IEEE Journal of Selected Topics in Signal Processing, vol. 10, no. 3, pp. 528-542, April 2016.

[18] C. Rusu, R. Mendez-Rial, N. Gonzalez-Prelcic, and R. Heath, "Low complexity hybrid precoding strategies for millimeter wave communication systems," IEEE Transactions on Wireless Communications, vol. PP, no. 99, pp. 1-1, 2016.

[19] R. Mndez-Rial, C. Rusu, A. Alkhateeb, N. Gonzlez-Prelcic, and R. W. Heath, "Channel estimation and hybrid combining for mmWave: Phase shifters or switches?" in Information Theory and Applications Workshop (ITA), 2015, Feb 2015, pp. 90-97.

[20] X. Yu, J. C. Shen, J. Zhang, and K. B. Letaief, "Alternating Minimization Algorithms for Hybrid Precoding in Millimeter Wave MIMO Systems," IEEE Journal of Selected Topics in Signal Processing, vol. 10, no. 3, pp. 485-500, April 2016.

[21] R. Mendez-Rial, C. Rusu, N. González-Prelcic, A. Alkhateeb, and R. W. Heath Jr, "Hybrid MIMO Architectures for Millimeter Wave Communications: Phase Shifters or Switches?" IEEE Access, vol. 4, pp. 247-267, January, 2016 2016. [Online]. Available: http://ieeexplore.ieee.org/stamp/stamp.jsp?tp=\&arnumber $=7370753$

[22] T. Lipp and S. Boyd, "Variations and extension of the convex-concave procedure," Optimization and Engineering, vol. 17, no. 2, pp. 263-287, 2016. [Online]. Available: http://dx.doi.org/10.1007/s11081-015-9294-x

[23] M. A. Vázquez, L. Blanco, A. Pérez-Neira, and M. A. Lagunas, "Phaseonly transmit beamforming for spectrum sharing microwave systems," in Smart Antennas (WSA), 2016 International ITG Workshop on, March 2016, pp. 1-8.

[24] M. R. Akdeniz, Y. Liu, M. K. Samimi, S. Sun, S. Rangan, T. S. Rappaport, and E. Erkip, "Millimeter Wave Channel Modeling and Cellular Capacity Evaluation," IEEE Journal on Selected Areas in Communications, vol. 32, no. 6, pp. 1164-1179, June 2014.

[25] A. Maltsev and et al, "D5.1 - Channel Modeling and Characterization," MiWEBA Project (FP7-ICT-608637), Public Deliverable, Jan. 2014.
[26] R. Lopez-Valcarce, N. Gonzalez-Prelcic, C. Rusu, and R. W. Heath, "Hybrid Precoders and Combiners for mmWave MIMO Systems with Per-Antenna Power Constraints," in 2016 IEEE Global Communications Conference (GLOBECOM), Dec 2016, pp. 1-6.

[27] O. Mehanna, K. Huang, B. Gopalakrishnan, A. Konar, and N. D. Sidiropoulos, "Feasible Point Pursuit and Successive Approximation of Non-Convex QCQPs," IEEE Signal Processing Letters, vol. 22, no. 7, pp. 804-808, July 2015.

[28] Z. q. Luo, W. k. Ma, A. M. c. So, Y. Ye, and S. Zhang, "Semidefinite Relaxation of Quadratic Optimization Problems," IEEE Signal Processing Magazine, vol. 27, no. 3, pp. 20-34, May 2010.

[29] G. R. Lanckriet and B. K. Sriperumbudur, "On the Convergence of the Concave-Convex Procedure," in Advances in Neural Information Processing Systems 22, Y. Bengio, D. Schuurmans, J. D. Lafferty, C. K. I. Williams, and A. Culotta, Eds. Curran Associates, Inc., 2009, pp. 1759-1767. [Online]. Available: http://papers.nips.cc/paper/3646on-the-convergence-of-the-concave-convex-procedure.pdf

[30] M. S. Lobo, L. Vandenberghe, S. Boyd, and H. Lebret, "Applications of second-order cone programming," Linear Algebra and its Applications, vol. 284, no. 13, pp. 193 - 228, 1998, international Linear Algebra Society (ILAS) Symposium on Fast Algorithms for Control, Signals and Image Processing. [Online]. Available: http://www.sciencedirect.com/science/article/pii/S0024379598100320 